\title{
Investigación universitaria, clima y cultura organizacional en la Universidad César Vallejo - sede Lima Este - SJL
}

\author{
Dr. Johnny Félix Farfán-Pimentel \\ felix13200@hotmail.com \\ https://orcid.org/0000-0001-6109-4416 \\ Universidad César Vallejo \\ Dr. Raúl Delgado-Arenas \\ rdelgadoa@ucv.edu.pe \\ https://orcid.org/0000-0003-4941-4717 \\ Universidad César Vallejo \\ Dr. Rommel Lizandro-Crispín \\ rolic451@gmail.com \\ https://orcid.org/0000-0003-1091-225X \\ Universidad César Vallejo \\ Dra. Yolanda Josefina Huayta-Franco \\ yolandahuaytafranco2014@gmail.com \\ https://orcid.org/0000-0003-0194-8891 \\ Universidad César Vallejo \\ Mtra. Diana Eulogia Farfán-Pimentel \\ diana75_farfan@hotmail.com \\ https://orcid.org/0000-0002-1555-1919 \\ Universidad César Vallejo
}

\section{RESUMEN}

La investigación que se realizó tuvo como propósito esencial estudiar la investigación universitaria, el cima y cultura organización como factores que inciden directamente en el proceso formativo de los estudiantes universitarios de la facultad de ingeniería de la Universidad César Vallejo; siendo está muy necesaria ya que la preparación de los futuros profesionales se sostengan sobre una sólida base científica y el desarrollo de una actitud investigativa que propenda la generación de nuevos conocimientos y que los hallazgos encontrados reafirmen la motivación e interés en los estudiantes y de parte de las autoridades universitarias el fomento y promoción de investigaciones que contribuyan a la mejora de la calidad de vida de la población en su conjunto. Siendo el tipo de estudio básico, de nivel 
descriptivo y correlacional, de diseño no experimental se aplicó tres instrumentos para la recolección de datos y la confiabilidad Alfa de Cronbach fueron para la variable investigación universitaria (alfa=0,939); clima organizacional (alfa $=0,835$ ) y cultura organizacional (alfa=0,947). Se evidenció las correlaciones estadísticas entre las variables investigación universitaria y clima organizacional con una rho $=0,442$ y p $=0,000$ mientras que para investigación universitaria y cultura organizacional con una rho=0,328 y p=0,000.

Palabras clave: investigación universitaria; clima organizacional; cultura organizacional. 


\title{
University research, climate and organizational culture in the Universidad César Vallejo - Lima East campus - SJL
}

\begin{abstract}
The essential purpose of the research was to study university research, the summit and organizational culture as factors that directly affect the formative process of university students in the Faculty of Engineering at the Universidad César Vallejo; It is necessary for the preparation of future professionals to be sustained on a solid scientific basis and the development of a research attitude that favors the generation of new knowledge and that the findings reaffirm the motivation and interest of the students and on the part of the university authorities to encourage and promote research that contributes to the improvement of the quality of life of the population as a whole. Being the basic type of study, of descriptive and correlational level, of non-experimental design, three instruments were applied for data collection and the Cronbach's Alpha reliability were for the university research variable (alpha $=0.939)$; organizational climate (alpha $=0.835)$ and organizational culture (alpha=0.947). Statistical correlations were evidenced between the variables university research and organizational climate with an rho $=0.442$ and $\mathrm{p}=0.000$ while for university research and organizational culture with an rho $=0.328$ and $\mathrm{p}=0.000$.
\end{abstract}

Key words: university research, organizational climate, organizational culture.

Artículo recibido: 20 diciembre. 2021 Aceptado para publicación: 10 enero 2022 Correspondencia: felix13200@hotmail.com Conflictos de Interés: Ninguna que declarar 


\section{INTRODUCCIÓN}

En el contexto actual la investigación científica ocupa un lugar primordial en el quehacer de la universidad posibilitando el desarrollo de conocimientos e innovación tecnológica en circunstancias en la que la población demanda la solución a los más álgidos problemas que debe enfrentar; es por ello que, existe la necesidad de ahondar y priorizar la actividad científica como una fuente de generación de alternativas de solución en los distintos campos del conocimiento científico y que la autoridades universitarias tienen una gran responsabilidad y compromiso de impulsar e implementar a partir de sus laboratorios, recursos tecnológicos y ámbitos de investigación con miras a propiciar cambios esenciales en la comunidad universitaria. En ese sentido, la investigación científica es la vía que orienta el progreso en el conocimiento de la realidad, a través del empleo del método científico que impele la obtención de información de carácter relevante y demostrable que permite la comprensión, verificación y aplicación de teorías científicas; en ese conjunto de ideas muestra características como la reflexividad, sistematicidad y metodológica en el proceso de trabajo científico de la comunidad universitaria. Asimismo, la investigación científica viene a ser una búsqueda de nuevos conocimientos y alternativas de solución a las múltiples situaciones problemáticas, para ello el método científico señala la trayectoria a seguir en el proceso indagatorio y los procedimientos que se revelan de cómo proceder; estas incluyen técnicas, instrumentos y mecanismos eficaces para dar a conocer los hallazgos encontrados experimentalmente y teóricamente. En tal sentido la educación universitaria es la viga maestra para erigir una sociedad justa del conocimiento, adicionado a ello con el impacto respectivo en la economía de una nación; es arduo el trabajo en la universidad teniendo en cuenta las posibilidades del potencial humano en la adquisición de habilidades y competencias para la formación de cuadros de especialistas altamente capacitados para brindar las mejores soluciones a la nación. Para ello se realizará un análisis del estado del arte en base a los constructos de la pesquisa ejecutada, a continuación, se detallan los antecedentes empleados:

Vargas (2020) en su tesis doctoral tuvo como propósito el estudio de la actitud hacia la investigación científica. Señaló que, en la actualidad la investigación científica enfrenta enormes retos que resolver en ese sentido la función sustancial y de carácter obligatoria en el sistema universitario es la de fomentar en la comunidad universitaria a través de la producción intelectual de conocimientos que respondan a las grandes demandas de la sociedad en los múltiples campos del quehacer humano. La investigación universitaria es un 
conjunto de procesos fundamentales las cuales forja en los universitarios el desarrollo del pensamiento crítico-reflexivo que posibilita la generación de nuevas perspectivas investigativas, de tecnología de punta, de desarrollo de modelos híbridos en su globalidad. Los resultados mostraron que $\mathrm{X}^{2}($ Calculada $)=9,27$ y $\mathrm{X}^{2}$ (Tabla) $=3,84$ Por lo tanto $\mathrm{X}^{2}$ $($ Calculada $)=9,27>X^{2}($ Tabla $)=3,84$; para un p-valor $=0,000<0,05$. Se concluyó que los docentes de universidades de Huancayo manifiestan características de una cualidad original hacia el trabajo académico de la investigación científica y presentan una actitud positiva hacia la investigación, en los tres componentes: afectivo, cognitivo y conductual.

Dáher, Panunzio y Hernández (2018) en su pesquisa señalaron que la investigación es la base esencial en el desarrollo de la sociedad y ofrece una gama muy importante de soluciones desde la perspectiva científica, tecnológica, socioeducativa y humanística; es pues gracias a los descubrimientos es posible contar con una mirada multidisciplinar de la problemática de los aspectos más variados y multiformes; por cuanto en conjunción a la docencia universitaria y la proyección a la comunidad forman los pilares básicos para la construcción de nuevos conocimientos, en consecuencia, las universidades conforman el núcleo que garantizará los logros científico-tecnológico de un país y son los principales motores que impulsarán los grandes cambios en la sociedad actual.

Bohórquez (2015) en la tesis doctoral tiene como propósito identificar la relación entre la actitud científica y el logro de competencias del curso de investigación en universitarios. El tipo de investigación fue básica, de diseño no experimental, siendo la población de 620 y una muestra de 237 universitarios. En ese sentido, la actitud científica juega un papel fundamental en el desarrollo de las capacidades y habilidades de los estudiantes universitarios, ya que, los escenarios en las que hay que intervenir muestran un conjunto de características disímiles; en el contexto socioeducativo se pretende desarrollar un conjunto de experiencias de aprendizaje que posibilite la comprensión de la realidad desde un enfoque analítico y crítico que responda a las interrogantes y sean vinculantes con los contextos de la cotidianeidad y ampliar las fronteras del conocimiento. Los resultados que se obtuvo fueron que el estadístico $\mathrm{r}$ de Pearson se evidenció una correlación entre la actitud científica y el logro de competencias $(\mathrm{r}=0,600)$, en conceptuales $(\mathrm{r}=0,545)$, en procedimentales $(\mathrm{r}=0,584)$ y en actitudinales $(\mathrm{r}=0,523)$. Se concluyó que la actitud científica influye de manera positiva en el logro de competencias.

Támara (2018), en la tesis de maestría tuvo como finalidad estudiar el clima organizacional y la calidad educativa. La investigación básica de nivel descriptivo y correlacional siendo el 
diseño no experimental, la muestra fue de 35 docentes. El clima organizacional es un factor decisivo en el proceso formativo de los estudiantes, debido a que se sustenta en una adecuada convivencia estructurada sobre la base de una buena comunicación y se organizan las actividades de aprendizaje en función a las necesidades de aprendizaje de los educandos, se pretende a través del clima organizacional fortalecer las capacidades cognitivas para poder afrontar los retos en una sociedad cada vez más compleja y que se requiere de sustentos éticos y valorativos en la organización educativa. Los resultados muestran que existe una asociación directa y significativa entre el clima organizacional y la calidad educativa (rho=0,818 y $\mathrm{p}=0,000$ ). Se concluyó que el clima organizacional incide de manera significativa con la calidad educativa ya que esta propicia un ambiente de armonía y respeto entre sus integrantes de la comunidad educativa.

Pérez (2012) en la pesquisa tiene como propósito el estudio entre el clima institucional y desempeño docente. La investigación fue básica de nivel descriptivo y correlacional y de diseño no experimental, la muestra fue de 100 docentes y se aplicó un cuestionario para el recojo de información. El clima en la organización educativa se concibe como la percepción que se tiene a nivel de la institución educativa como el nivel de comunicación, prevalencia de valores entre los integrantes de la comunidad escolar que posibilita el desarrollo de actividades y procesos educativos en virtud que incide en el reconocimiento de normas de convivencia y el respeto en el quehacer educativo y estos se reflejaran a nivel de las actitudes de los estudiantes y docentes. En ese sentido, un buen nivel en la interacción responsable, una atmósfera adecuada y una sensación que favorece el nivel de entendimiento implica que el clima es beneficioso para la acción pedagógica de los docentes. Los resultados demuestran según el coeficiente $r$ de Pearson que existe una correlación entre el clima y el desempeño en $r=0,46$; con la comunicación $r=0,88$; con la motivación $r=0,95$; con la confianza $r=0,94$ y la participación $r=0,94$. Se concluyó que el clima es un factor determinante para el desempeño de los profesores y afecta en la motivación y participación de manera sustancial. Mendoza (2011), en la tesis de maestría el objetivo fue identificar la relación entre el clima institucional y el desempeño docente. En la pesquisa se empleó el enfoque cuantitativo, se aplicó dos cuestionarios con la escala Likert. La investigación fue de tipo descriptivo y correlacional de corte transversal. El clima es la escuela es un factor que contribuye al logro de los objetivos planificados y que a través de una adecuada gestión organizacional y visto el cumplimiento de las normas establecidas posibilitaran alcanzar las características deseables entre sus integrantes. Según las indagaciones realizadas queda claro la importancia 
de entender el nivel de interacciones entre las personas y el comportamiento que demuestran en el ámbito específico y que afectaría el desarrollo de las actividades educativas; es por ello que, el clima en la escuela tiene una resultante que incide directamente en los procesos educativos y las condiciones ambientales características de la institución determinando sustancialmente el nivel de calidad y productividad de docentes y estudiantes. Los resultados fueron según la rho de Spearman las correlaciones entre el clima institucional y desempeño docente (rho=0,537); con comportamiento institucional (rho=0,957); con capacidad organizacional $(\mathrm{rho}=0,890)$ y dinámica institucional $(\mathrm{rho}=0,920)$. Se concluyó que el clima institucional favorece positivamente el desempeño de los docentes y contribuye el logro de los objetivos estratégicos de la escuela.

Saccsa (2010), en la tesis de maestría, tuvo la finalidad de estudiar el clima institucional y el desempeño docente. El tipo fue básico de diseño no experimental y de corte transversal. El clima en una institución escolar es determinante para contar con un ambiente que posibilite el desarrollo de los educandos; y en donde los estilos comunicativos juegan un papel crucial para el logro del perfil en los educandos, es por ello que, la escuela debe promover una comunicación y la participación permanente proponiendo la práctica de valores éticos entre los miembros de la comunidad educativa. En ese sentido el clima que se experimenta en la organización educativa es la búsqueda de un continuo proceso de mejoramiento del ambiente en la que se perciba un nivel de respeto y adecuada comunicación. Los resultados obtenidos en la regresión logística se obtuvo un valor $\mathrm{R}=0,768 \mathrm{p}=0,000$. Se concluyó que el clima institucional afecta el desempeño de la escuela de manera ostensible y se debe de trabajar en este aspecto con miras a elevar el mejoramiento de las relaciones humanas en la comunidad educativa.

\section{Definición de investigación universitaria}

Ríos (2013), señaló que la investigación científica tiene como propósito la interpretación de los hechos o fenómenos naturales que se expresan a través de leyes que permiten la comprensión de las manifestaciones de la naturaleza; así también proporcionan un sustento científico que se aplica a la solución de problemas, es que a partir de las reflexiones cognitivas se puede brindar un aporte esencial para la comunidad de investigadores. En ese sentido, la investigación científica fundamenta su actividad mediante la aplicación del método científico para verificar y contrastar las hipótesis de campo, siendo estas esenciales en la labor investigativa. En ese sentido, Duarte (2015), indicó que la investigación científica se establece como uno de los pilares esenciales en las actividades académicas en las 
universidades; es por ello que, la importancia que esta tienen en la generación de conocimientos que se logran inevitablemente a través de la investigación e innovación tecnológica las cuales constituyen la base para la construcción de una infraestructura de carácter productivo, social, científico y tecnológico para las instituciones universitarias. No obstante, se debe entender que la actividad científica es realizada por los docentes investigadores comprometidos con la transformación de la cultura y la sociedad en su conjunto. Por consiguiente, Naupas (2009), indicó que la investigación científica en la universidad se constituye como parte del desarrollo de conocimientos y que impulsa el progreso social a través de la actividad descubridora de hallazgos, de verificación y contrastación de hipótesis de trabajo de la labor investigadora y se garantice el avance de la ciencia en el contexto nacional

Figura 1. ¿Qué es Investigar?

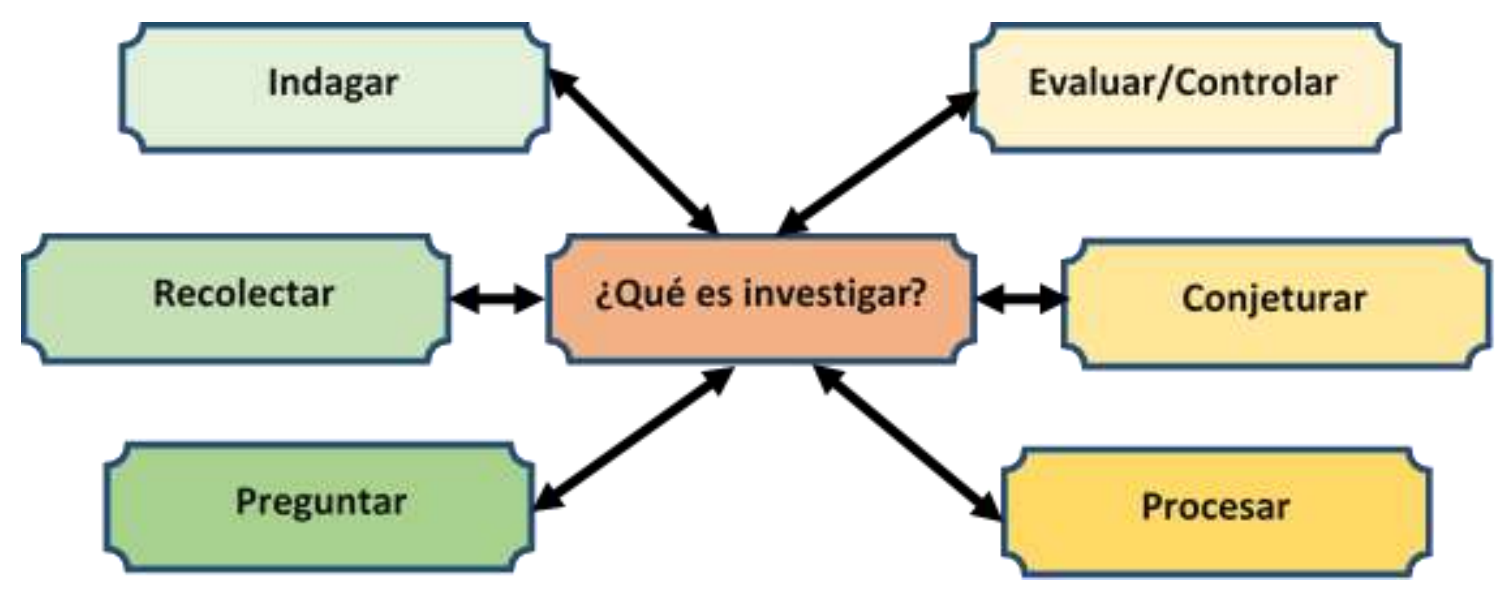

\section{Los procesos de la investigación científica}

En todo proceso de investigación se puede apreciar la existencia de factores de naturaleza interna como externa, en el aspecto interno está referida básicamente a la dinámica esencial y sus propiedades intrínsecas como sistema complejo; a su vez, los de índole externo están relacionados con condicionantes que ejercen un determinado grado de influencia sobre lo interno de la investigación científica. Es por ello que, Piscoya (1987) indicó que los procesos de investigación científica presentan determinados aspectos como: (a) Lo epistémico, se fundamenta en el mecanismo de la lógica científica, los aspectos formales y contenidos que posibilitan el establecimiento de la verdad científica; (b) Lo social, esta se basa en las condiciones de naturaleza histórica, cultural y económica a la que está ligada la investigación 
científica; y (c) Lo psicológico, se sustenta en la experiencia personal del investigador, que se tipifican como factores de orden subjetivo, se tornan irrelevantes en la construcción del conocimiento científico que es demostrable.

Figura 2. Aspectos de la investigación científica

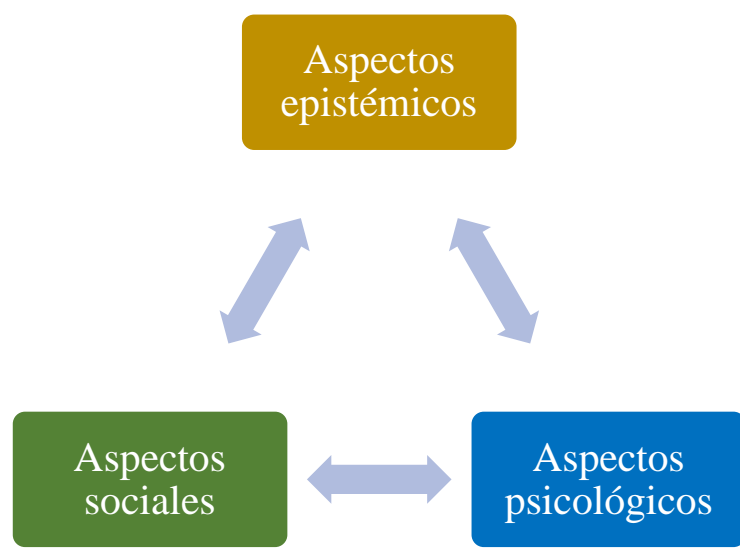

\section{Clima organizacional}

Chiavenato (1999), indicó que al respecto que el clima organizacional es la que se percibe o refleja en función a las necesidades organizativas y las personas que conforman la organización, es por ello que, una organización se estructura en varios niveles de responsabilidad que cumplen una determinada tarea para el logro de los objetivos institucionales. Entre tanto, Chiang, Núñez y Martín (2010) señalaron que el factor de clima organizacional es crucial para la buena marcha de la organización y esta considera como una de las fortalezas a nivel de las instituciones, es un hecho que se basa esencialmente en la fluidez de la comunicación en los entornos organizativos y que orienta a alcanzar los propósitos prestablecidos por la gestión organizacional. En ese sentido, Martín (1999) precisó que el clima organizacional se establece en un conjunto de cualidades, atributos, características o propiedades en el contexto de un ambiente de trabajo específico, estas son percibidas o experimentadas por los integrantes de la organización y que inciden en el comportamiento de los colaboradores de una institución. Por consiguiente, Méndez (2006) acotó que es la resultante en la manera como los individuos establecen procesos de interacción entre pares y esta se encuentra influenciada por el sistema de valores, las actitudes, las creencias y el ambiente interno organizacional.

\section{Importancia del clima organizacional}

Al respecto, Guevara (2018) sostuvo que la gestión del clima organizacional, constituye en 
estos momentos el compromiso y la responsabilidad que se comparte con las personas que gestionan las organizaciones y el potencial humano que se desarrolla en la organización; en ese sentido, el clima organizacional que muestra un adecuado nivel de interacción entre sus componentes posibilitaría generar un auspicioso nivel de eficiencia de la gestión y la satisfacción de los colaboradores en el desempeño de las acciones propias de la responsabilidad creando un ambiente positivo para el logro de objetivos estratégicos. Por consiguiente, en el estudio del clima organizacional en el contexto educacional adquiere relevancia ya que se muestra como un factor sustancial para lograr el desarrollo de la entidad y esta repercutirá en la visión integradora globalizadora y realista en el proceso de construcción de un ambiente motivador y que favorezca alcanzar las metas propuestas. Asi también, Denison (2006) manifestó que el clima organizacional se entiende como la cualidad del ambiente interno y que es percibida por los integrantes de la organización; es por ello que, la importancia de dicho constructo fortalecerá los valores y afectará en consonancia con las actitudes de los colaboradores que a su vez se reflejará en los niveles de productividad de la organización.

\section{Figura 3. Características del clima organizacional}

Situación: Tiene que ver con el lugar el trabajo de la organización.

Permanencia: A pesar de experimentar cambios por situaciones coyunturales.

Impacto: Sobre los comportamientos de los miembros de la institución.

Compromiso: Afecta el grado de compromiso e identificación de los miembros de la organización con ésta.

Comportamiento: Es afectado por los

comportamientos y actitudes de los miembros de

la organización

Estructuras: Tales como estilo de dirección,

políticas y planes de gestión, sistemas de

contratación y despidos, etc. 


\section{Cultura organizacional}

El proceso de investigación universitaria se nutre sobre la base del esfuerzo y dedicación de los miembros de la comunidad universitaria en la que enfrentan a retos de manera constante en pos de alcanzar resultados valiosos para el avance en las diversas áreas del conocimiento; a su vez, que se requiere contar con una red de investigadores que posibilite el intercambio fluido de información científica, en esta confluencia de ideas la cultura organizacional juega un importante papel; en ese sentido se compartes valores éticos, ideales, conocimientos y todo aquello que es de valía en la construcción de aportes teóricos y de innovación tecnológica. En la actualidad las universidades necesitan realizar grandes cambios tanto en el aspecto estructural como le funcional; en virtud de ello, se deben desarrollar habilidades gerenciales que atienda a favorecer factores como el liderazgo, la comunicación y los modelos comportamentales, en tal sentido, es necesario ahondar es aspectos como el clima y la cultura organizacionales y su incidencia en los procesos investigativos. Es por ello que, Fuentes (2012), señaló que la cultura organizacional está conformada por la cultura formal las cuales se expresan a través de las actitudes, los comportamientos, los valores y creencias de los participantes, en tanto que lo informal por los comportamientos reales. Asimismo, Peniche (2012) recalcó que, la cultura organizacional se establece por los valores y significados claramente constituidos e involucra acciones y propósitos validados consensuadamente. Los investigadores Yopan, Palmero y Santos (2020), manifestaron que la cultura organizacional se considera en estos momentos en uno de los activos más significativos en una organización y las teorías que fortalecen este constructo se encuentra en los campos administrativos, organizacionales y comunicacionales; en ese sentido, la cultura organizacional se construye a través de procesos de interacción e interpretación, en esta argumentación se configura en una tarea que remarca complejidad y de carácter cualitativo.

\section{Problema general}

- ¿Qué relación existe entre la participación de los estudiantes de la Facultad de Ingeniería en la Investigación universitaria, el clima y la cultura organizacional en la Universidad César Vallejo - sede Lima Este?

\section{Problemas específicos}

- ¿Qué relación existe entre la participación de los estudiantes de la Facultad de Ingeniería en los procesos de enseñanza de la Investigación universitaria, el clima y la cultura organizacional en la Universidad César Vallejo - sede Lima Este? 
- ¿Qué relación existe entre la participación de los estudiantes de la Facultad de Ingeniería en los procesos de aprendizaje de la Investigación universitaria, el clima y la cultura organizacional en la Universidad César Vallejo - sede Lima Este?

- ¿Qué relación existe entre la participación de los estudiantes de la Facultad de Ingeniería en el desarrollo de proyectos de investigación, el clima y la cultura organizacional en la Universidad César Vallejo - sede Lima Este?

\section{Objetivo general}

- Determinar la relación que existe entre la participación de los estudiantes de la Facultad de Ingeniería en la Investigación universitaria, el clima y la cultura organizacional en la Universidad César Vallejo - sede Lima Este.

\section{Objetivos específicos}

- Determinar la relación que existe entre la participación de los estudiantes de la Facultad de Ingeniería en los procesos de enseñanza de la Investigación universitaria, el clima y la cultura organizacional en la Universidad César Vallejo - sede Lima Este.

- Determinar la relación que existe entre la participación de los estudiantes de la Facultad de Ingeniería en los procesos de aprendizaje de la Investigación universitaria, el clima y la cultura organizacional en la Universidad César Vallejo - sede Lima Este.

- Determinar la relación que existe entre la participación de los estudiantes de la Facultad de Ingeniería en el desarrollo de proyectos de investigación, el clima y la cultura organizacional en la Universidad César Vallejo - sede Lima Este.

\section{Hipótesis general}

- Existe una relación significativa en la participación de los estudiantes de la Facultad de Ingeniería en la Investigación Universitaria, el Clima y Cultura Organizacional en la Universidad César Vallejo - Sede Lima Este.

\section{Hipótesis específicas}

- El Clima y Cultura Organizacional se relacionan significativamente con la participación de los estudiantes de la Facultad de Ingeniería con los procesos de enseñanza de la Investigación Universitaria en la Universidad César Vallejo - sede Lima Este.

- El Clima y Cultura Organizacional se relacionan significativamente con la participación de los estudiantes de la Facultad de Ingeniería con los procesos de aprendizaje de la Investigación Universitaria en la Universidad César Vallejo - sede Lima Este.

- El Clima y Cultura Organizacional se relacionan significativamente con la participación de los estudiantes de la Facultad de Ingeniería en el desarrollo de proyectos de 
investigación en la Universidad César Vallejo - sede Lima Este.

\section{MÉTODO}

La investigación es de tipo básica, no experimental y de nivel descriptivo-correlacional; ya que aportará desde el plano teórico una perspectiva para la comprensión de la investigación, clima y cultura organizacionales en el desarrollo del trabajo académico de la comunidad universitaria; brindando alcances para una adecuada marcha de los objetivos estratégicos de la institución universitaria. Asi también se aplicarán métodos, técnicas e instrumentos que posibilitarán el recojo de información para posteriormente realizar el tratamiento, análisis y presentación de resultados.

\section{Operacionalización de las variables}

\section{Variable 1: Investigación universitaria.}

\section{Definición conceptual}

Ñaupas (2009), indicó que la investigación científica en la universidad se constituye como parte del desarrollo de conocimientos y que impulsa el progreso social a través de la actividad descubridora de hallazgos, de verificación y contrastación de hipótesis de trabajo de la labor investigadora y se garantice el avance de la ciencia en el contexto nacional.

\section{Tabla 1}

Operacionalización de la variable 1: Investigación universitaria

\begin{tabular}{|c|c|c|c|c|}
\hline Dimensiones & Indicadores & Ítems & $\begin{array}{l}\text { Escala / } \\
\text { Valores }\end{array}$ & $\begin{array}{c}\text { Niveles / } \\
\text { Rangos }\end{array}$ \\
\hline $\begin{array}{l}\text { Procesos de } \\
\text { enseñanza }\end{array}$ & $\begin{array}{l}\text { Naturaleza colectiva } \\
\text { Función Social } \\
\text { Carácter gestionario, } \\
\text { temporal. }\end{array}$ & $\begin{array}{l}1,2 \\
3,4 \\
5,6 \\
7,8 \\
9,10\end{array}$ & & \\
\hline $\begin{array}{l}\text { Procesos de } \\
\text { aprendizaje }\end{array}$ & $\begin{array}{l}\text { Base tecnológica, } \\
\text { infraestructural } \\
\text { Crecimiento por } \\
\text { Aprendizajes } \\
\text { Comportamientos } \\
\text { regulados. }\end{array}$ & $\begin{array}{l}11,12 \\
13,14 \\
15,16 \\
17,18 \\
19,20\end{array}$ & $\begin{array}{c}\text { Escala } \\
\text { ordinal: } \\
\text { Likert } \\
\text { Nunca (1) } \\
\text { Casi nunca } \\
\text { (2) }\end{array}$ & $\begin{array}{c}\text { Deficiente } \\
\text { [10-23] } \\
\text { Moderado } \\
\text { [24-37] } \\
\text { Eficiente } \\
\text { [38-50] }\end{array}$ \\
\hline $\begin{array}{l}\text { Participación } \\
\text { en proyectos } \\
\text { de } \\
\text { investigación }\end{array}$ & $\begin{array}{l}\text { Carácter comunicacional } \\
\text { Liderazgo } \\
\text { Motivación } \\
\text { Toma de decisiones }\end{array}$ & $\begin{array}{l}21,22 \\
23,24 \\
25,26 \\
27,28 \\
29,30\end{array}$ & $\begin{array}{l}\text { A veces (3) } \\
\text { Casi siempre } \\
\qquad(4) \\
\text { Siempre (5) }\end{array}$ & \\
\hline
\end{tabular}




\section{Variable 2: Clima organizacional.}

\section{Definición conceptual}

Chiavenato (1999), indicó que al respecto que el clima organizacional es la que se percibe o refleja en función a las necesidades organizativas y las personas que conforman la organización, es por ello que, una organización se estructura en varios niveles de responsabilidad que cumplen una determinada tarea para el logro de los objetivos institucionales.

\section{Tabla 2}

Operacionalización de la variable 2: Clima organizacional

\begin{tabular}{|c|c|c|c|c|}
\hline Dimensiones & Indicadores & Ítems & $\begin{array}{l}\text { Escala / } \\
\text { Valores }\end{array}$ & $\begin{array}{c}\text { Niveles / } \\
\text { Rangos }\end{array}$ \\
\hline $\begin{array}{l}\text { Organización } \\
\text { institucional }\end{array}$ & $\begin{array}{l}\text { Percepción } \\
\text { global } \\
\text { Organización } \\
\text { Planificación } \\
\text { Evaluación }\end{array}$ & $\begin{array}{c}1,2 \\
3,4 \\
5,6 \\
7,8 \\
9,10\end{array}$ & & \\
\hline $\begin{array}{l}\text { Relaciones } \\
\text { humanas }\end{array}$ & $\begin{array}{l}\text { Capacidades } \\
\text { Habilidades } \\
\text { Destrezas }\end{array}$ & $\begin{array}{l}11,12 \\
13,14 \\
15,16 \\
17,18 \\
19,20\end{array}$ & $\begin{array}{c}\text { Escala } \\
\text { ordinal: } \\
\text { Likert } \\
\text { Nunca (1) } \\
\text { Casi nunca }\end{array}$ & $\begin{array}{c}\text { Deficiente } \\
\text { [10-23] } \\
\text { Moderado } \\
\text { [24-37] } \\
\text { Eficiente }\end{array}$ \\
\hline $\begin{array}{l}\text { Clima } \\
\text { institucional }\end{array}$ & $\begin{array}{l}\text { Respeto } \\
\text { Buen trato } \\
\text { Comunicación }\end{array}$ & $\begin{array}{l}21,22 \\
23,24 \\
25,26 \\
27,28 \\
29,30\end{array}$ & $\begin{array}{c}(2) \\
\text { A veces (3) } \\
\text { Casi siempre } \\
\quad(4) \\
\text { Siempre (5) }\end{array}$ & [38-50] \\
\hline
\end{tabular}

\section{Variable 3: Cultura organizacional.}

\section{Definición conceptual}

Peniche (2012) recalcó que, la cultura organizacional se establece por los valores y significados claramente constituidos e involucra acciones y propósitos validados consensuadamente 


\section{Tabla 3}

Operacionalización de la variable 3: Cultura organizacional

\begin{tabular}{|c|c|c|c|c|}
\hline Dimensiones & Indicadores & Ítems & Escala / Valores & $\begin{array}{c}\text { Niveles / } \\
\text { Rangos }\end{array}$ \\
\hline \multirow{5}{*}{$\begin{array}{l}\text { Claridad de } \\
\text { metas }\end{array}$} & Propósitos & 1,2 , & & \\
\hline & Objetivos & 3,4 & & \\
\hline & Metas establecidas & 5,6 & & \\
\hline & \multirow[t]{2}{*}{ Organización } & 7,8 & & \\
\hline & & 9,10 & & \\
\hline \multirow{8}{*}{ Coordinación } & Trabajo de equipo & 11,12 & & \\
\hline & Logros académicos & 13,14 & Escala ordinal: & Deficiente \\
\hline & Procesos & 15,16 & Likert & {$[10-23]$} \\
\hline & curriculares & 17,18 & Nunca (1) & Moderado \\
\hline & Procesos & 19,20 & Casi nunca (2) & [24-37] \\
\hline & didacticos & & A veces (3) & Eficiente \\
\hline & \multirow{2}{*}{ pedagógicos } & & Casi siempre (4) & {$[38-50]$} \\
\hline & & & Siempre (5) & \\
\hline \multirow{5}{*}{$\begin{array}{l}\text { Resolución de } \\
\text { conflictos }\end{array}$} & Manejo & 21,22 & & \\
\hline & conflictos & 23,24 & & \\
\hline & Estrategias & 25,26 & & \\
\hline & solución & 27,28 & & \\
\hline & $\begin{array}{l}\text { Procesos } \\
\text { solución }\end{array}$ & 29,30 & & \\
\hline
\end{tabular}

\section{Población y muestra}

Arias, Villasís, Miranda (2016) indicaron que la población está constituida por un conjunto de elementos que reúnen características específicas y que cumple con una serie de determinados criterios de selección. Para el estudio la población fueron estudiantes de la facultad de ingeniería, siendo la muestra de 120 estudiantes que cursan la carrera profesional de ingeniería en la sede de Lima Este.

\section{Técnica e instrumento}

\section{Técnica}

El empleó de la técnica es un procedimiento que se emplea para la obtención de información, en ese sentido en el proceso de estructuración de la investigación hay que prever de qué manera se realizará el recojo de la información que será en insumo para el análisis de datos. Es por ello que, la técnica esta intrínsecamente relacionada con el método de investigación y responde a la perspectiva tanto teorética como filosófica del investigador (Sánchez y Reyes, 2015). 


\section{Instrumento}

El instrumento de investigación, posibilita la recolección de datos y esta se estructura en base a las variables de estudio, reúne una serie de requisitos que le brindan la potencia para el proceso de recojo de datos; a su vez debe poseer algunas propiedades básicas con la validez, confiabilidad y objetividad; es por ello que un instrumento es valioso en el proceso investigativo. En ese sentido, un instrumento de investigación toma como base una escala de medición y se emplean por lo general para ponderar actitudes y opiniones dependiendo el tipo de estudio a realizar (Sánchez y Reyes, 2015). Los resultados de fiabilidad de los instrumentos arrojaron para la variable investigación universitaria (alfa=0,939); clima organizacional (alfa=0,835) y cultura organizacional (alfa=0,947), las cuales se consideran en un nivel de muy buena y es aplicable para el propósito de la investigación.

\section{RESULTADOS}

Nivel de la variable 1: Investigación universitaria

Tabla 4 Nivel de la variable investigación universitaria

\begin{tabular}{lcccc}
\hline & Frecuencia & \multicolumn{1}{c}{ Porcentaje } & Porcentaje válido & Porcentaje acumulado \\
\hline Moderado & 8 & 6,7 & 6,7 & 6,7 \\
Eficiente & 112 & 93,3 & 93,3 & 100,0 \\
Total & $\mathbf{1 2 0}$ & $\mathbf{1 0 0 , 0}$ & $\mathbf{1 0 0 , 0}$ \\
\hline \multicolumn{4}{c}{ Fuente: base de datos }
\end{tabular}

Figura 4. Distribución porcentual según niveles de la variable: Investigación universitaria.

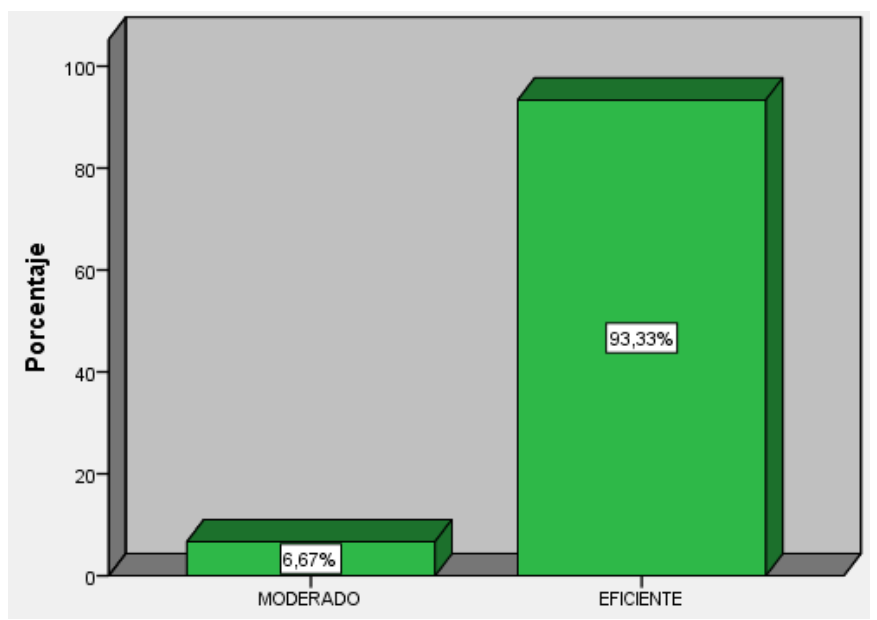

Nota: De la tabla 4 y figura 4 muestra según la percepción de encuestados que el nivel moderado de la variable se obtuvo un 6,67\% y el 93,33\% indica que es eficiente, lo que revela que la participación de la comunidad universitaria tiene claro los objetivos estratégicos que rige la política investigativa en la universidad en su conjunto. 


\section{Tabla 5}

Nivel de la variable clima organizacional

\begin{tabular}{lcccc}
\hline & Frecuencia & Porcentaje & Porcentaje válido & Porcentaje acumulado \\
\hline Regular & 12 & 10,0 & 10,0 & 10,0 \\
Bueno & 108 & 90,0 & 90,0 & 100,0 \\
Total & $\mathbf{1 2 0}$ & $\mathbf{1 0 0 , 0}$ & $\mathbf{1 0 0 , 0}$ \\
\hline \multicolumn{4}{c}{ Fuente: base de datos }
\end{tabular}

Figura 5. Distribución porcentual según niveles de la variable: Clima organizacional.

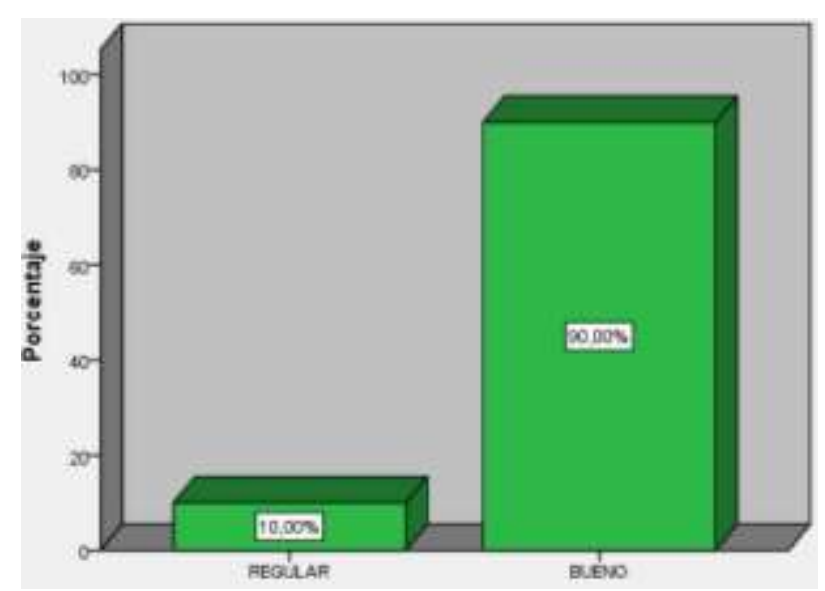

Nota: De la tabla 5 y figura 5 se percibe según los encuestados que la variable Clima organizacional alcanzó un 10,00\% situándose en un nivel regular mientras que el $90 \%$ asevera que se encuentra en un nivel bueno. En ese sentido, se observa que el clima organizacional juega un papel importante en el trabajo de investigación universitaria dado que en esta concurre una serie de elementos esenciales para la gestión investigativa.

\section{Tabla 6}

Nivel de la variable cultura organizacional

\begin{tabular}{lcccc}
\hline & Frecuencia & Porcentaje & Porcentaje válido & Porcentaje acumulado \\
\hline Promedio & 6 & 5,0 & 5,0 & 5,0 \\
Óptimo & 114 & 95,0 & 95,0 & 100,0 \\
Total & $\mathbf{1 2 0}$ & $\mathbf{1 0 0 , 0}$ & $\mathbf{1 0 0 , 0}$ & \\
\hline
\end{tabular}

Fuente: base de datos 
Figura 6. Distribución porcentual según niveles de la variable: Cultura organizacional.

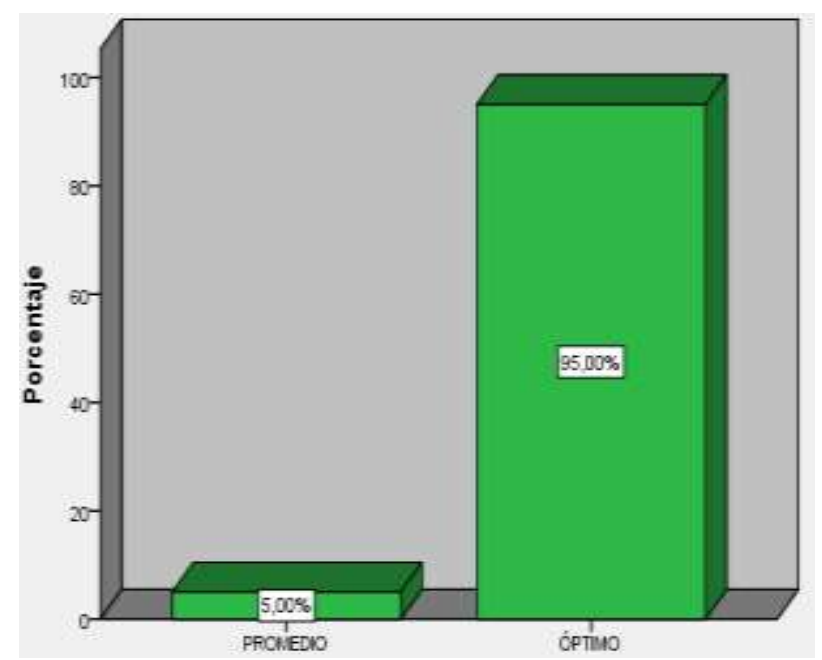

Nota: De la tabla 6 y figura 6 se percibe según los encuestados que la variable Cultura organizacional se obtuvo que un 5,00\% considera que se encuentra en un nivel promedio y el 90,00\% indica que es óptimo. Se observa que la cultura organizacional es el sustrato en la comunidad universitaria y que genera una comunicación vital para la puesta en marcha del propósito investigativos tanto en docentes como estudiantes universitarios.

\section{Contrastación de hipótesis.}

\section{Hipótesis general}

Ho: No existe una relación significativa entre el Clima y Cultura Organizacionales y la Investigación Universitaria en la Universidad César Vallejo - Sede Lima Este.

Hg: Existe una relación significativa entre el Clima y Cultura Organizacionales y la Investigación Universitaria en la Universidad César Vallejo - Sede Lima Este.

\section{Tabla 7}

Correlación de Clima y Cultura Organizacionales y la Investigación Universitaria

\begin{tabular}{lllcc}
\hline & & \multicolumn{2}{c}{$\begin{array}{c}\text { Investigación } \\
\text { Universitaria }\end{array}$} \\
\hline & & Coeficiente & de &, $442^{* *}$ \\
Rho de & Organizacional & correlación & &, 000 \\
Spearman & & $\mathrm{N}$ (bilateral) & &, 000 \\
& & Coeficiente & de & 120 \\
& Cultura & correlación & &, $328^{* *}$ \\
& Organizacional & Sig. (bilateral) & &, 040 \\
& & $\mathrm{~N}$ & & 120 \\
\hline
\end{tabular}

Fuente: base de datos 
De acuerdo a la tabla 7, el coeficiente de correlación de Spearman rho $=0,442 * *$ y $\mathrm{p}=0,000$ $<0,05$ entonces existe relación entre la variable Investigación Universitaria y el Clima organizacional y una rho $=0,328 * *$ con Cultura organizacional, siendo una correlación positiva moderada.

\section{Hipótesis específica 1.}

HE0: El Clima y Cultura Organizacionales se no relacionan significativamente con los procesos de enseñanza de la Investigación Universitaria en la Universidad César Vallejo - sede Lima Este.

HE1: El Clima y Cultura Organizacionales se relacionan significativamente con los procesos de enseñanza de la Investigación Universitaria en la Universidad César Vallejo - sede Lima Este.

\section{Tabla 8}

Correlación de Clima y Cultura Organizacionales y procesos de enseñanza

\begin{tabular}{lllc}
\hline & & \multicolumn{1}{c}{$\begin{array}{c}\text { Procesos de } \\
\text { enseñanza }\end{array}$} \\
\hline & Clima & $\begin{array}{l}\text { Coeficiente de } \\
\text { correlación }\end{array}$ &, $254^{* *}$ \\
\cline { 3 - 4 } Rho de & Organizacional & Sig. (bilateral) &, 005 \\
Spearman & & $\mathrm{N}$ & 120 \\
& & Coeficiente de &, $160^{*}$ \\
& Cultura & correlación &, 040 \\
& Organizacional & Sig. (bilateral) & 120 \\
& & $\mathrm{~N}$ & \\
\hline
\end{tabular}

Fuente: base de datos

De acuerdo a la tabla 8, el coeficiente de correlación de Spearman rho $=0,254 * *$ entonces existe relación entre los Proceso de enseñanza de la variable Investigación Universitaria y el Clima organizacional y una rho $=0,160 *$ con Cultura organizacional, es positiva baja.

\section{Hipótesis específica 2.}

HE0: El Clima y Cultura Organizacionales no se relacionan significativamente con los procesos de aprendizaje de la Investigación Universitaria en la Universidad César Vallejo - sede Lima Este.

HE2: El Clima y Cultura Organizacionales se relacionan significativamente con los procesos de aprendizaje de la Investigación Universitaria en la Universidad César Vallejo - sede Lima Este. 


\section{Tabla 9}

Correlación de Clima y Cultura Organizacionales y Procesos de aprendizaje

\begin{tabular}{lllcc}
\hline & & & $\begin{array}{c}\text { Procesos de } \\
\text { aprendizaje }\end{array}$ \\
\hline & Clima & $\begin{array}{l}\text { Coeficiente } \\
\text { correlación }\end{array}$ & de &, $481^{* *}$ \\
Rho de & Organizacional & Sig. (bilateral) & &, 000 \\
Spearman & & N & & 120 \\
& & Coeficiente & de &, $357^{* *}$ \\
& Cultura & correlación & &, 000 \\
& Organizacional & Sig. (bilateral) & &, 00 \\
\hline
\end{tabular}

Fuente: base de datos

De acuerdo a la tabla 9, el coeficiente de correlación de Spearman rho $=0,481 * *$ entonces existe relación entre los Procesos de aprendizaje de la variable Investigación Universitaria y el Clima organizacional y una rho $=0,357 * *$ con Cultura organizacional, es una correlación positiva moderada.

\section{Hipótesis específica 3.}

HE0: El Clima y Cultura Organizacionales no se relacionan significativamente con la participación en el desarrollo de proyectos de investigación en la Universidad César Vallejo - sede Lima Este.

HE3: El Clima y Cultura Organizacionales se relacionan significativamente con la participación en el desarrollo de proyectos de investigación en la Universidad César Vallejo - sede Lima Este.

\section{Tabla 10}

Correlación de Clima y Cultura Organizacionales y Participación en proyectos de investigación

\begin{tabular}{lllcc}
\hline & & & $\begin{array}{c}\text { Participación en } \\
\text { proyectos }\end{array}$ \\
\hline \multirow{4}{*}{$\begin{array}{llll}\text { Rho de } \\
\text { Spearman }\end{array}$} & Clima & $\begin{array}{l}\text { Coeficiente } \\
\text { correlación }\end{array}$ & de &, $547^{* *}$ \\
& & &, 000 \\
& Cultura & Sig. (bilateral) & & 120 \\
& Organizacional & Correlación & &, $467 * *$ \\
& & Sig. (bilateral) & &, 000 \\
& & N & \multicolumn{2}{c}{120} \\
\hline
\end{tabular}

Fuente: base de datos 
De acuerdo a la tabla 10, el coeficiente de correlación de Spearman rho $=0,547 * *$ entonces existe relación entre la Participación en proyectos de investigación y el Clima organizacional y una rho $=0,467 * *$ con Cultura organizacional, es correlación positiva moderada.

\section{DISCUSIÓN}

Vargas (2020) señaló que la investigación universitaria es un conjunto de procesos fundamentales las cuales forjan en los estudiantes el desarrollo del pensamiento críticoreflexivo siendo los resultados de $\mathrm{X}^{2}$ (Calculada) $=9,27$ y $\mathrm{X}^{2}$ (Tabla) $=3,84$ Por lo tanto $\mathrm{X}^{2}$ $($ Calculada $)=9,27>X^{2}($ Tabla $)=3,84 ;(p$-valor $=0,000<0,05)$, concluyendo que los docentes universitarios manifiestan características hacia el trabajo académico de la investigación científica y presentan una actitud positiva hacia la investigación, en los tres componentes: afectivo, cognitivo y conductual. Asimismo, Dáher, Panunzio y Hernández (2018) expresaron que la investigación es la base esencial para el desarrollo de la sociedad y ofrece una gama muy importante de soluciones desde la perspectiva científica, tecnológica, socioeducativa y humanística; en conjunción a la docencia universitaria y la proyección a la comunidad forman los pilares básicos para la construcción de nuevos conocimientos, siendo los principales motores que impulsarán los grandes cambios en la sociedad actual. En ese sentido, Bohórquez (2015) manifestó que, la actitud científica juega un papel fundamental en el desarrollo de las capacidades y habilidades de los estudiantes universitarios, en el contexto socioeducativo y que se pretende desarrollar un conjunto de experiencias de aprendizaje que posibilitará la comprensión de la realidad desde un enfoque analítico y crítico se obtuvo que el estadístico $r$ de Pearson una correlación entre la actitud científica y el logro de competencias $(r=0,600)$, en conceptuales $(r=0,545)$, en procedimentales $(\mathrm{r}=0,584)$ y en actitudinales $(\mathrm{r}=0,523)$. Asi también, Támara (2018) señaló que el clima organizacional es un factor decisivo en el proceso formativo de los estudiantes, y se sustenta en una adecuada convivencia estructurada sobre la base de una buena comunicación y se organizan las actividades de aprendizaje en función a las necesidades de aprendizaje de los educandos, se muestra que existe una asociación directa y significativa entre el clima organizacional y la calidad educativa ( $\mathrm{rho}=0,818$ y $\mathrm{p}=0,000)$. Consecuentemente, Pérez (2012) señaló que, el clima en la organización educativa se concibe como la percepción que se tiene a nivel de la institución educativa como el nivel de comunicación, prevalencia de valores entre los integrantes de la comunidad escolar que posibilita el desarrollo de actividades y procesos educativos, un buen nivel en la interacción responsable, una atmósfera adecuada y una sensación que favorece el nivel de entendimiento implica que el 
clima es beneficioso para la acción pedagógica de los docentes; los resultados según el coeficiente $r$ de Pearson que indica que existe una correlación entre el clima y el desempeño en $r=0,46$; con la comunicación $r=0,88$; con la motivación $r=0,95$; con la confianza $r=0,94$ y la participación r=0,94. Asimismo, Mendoza (2011) expresó que, el clima es la escuela es un factor que contribuye al logro de los objetivos planificados y que a través de una adecuada gestión organizacional y visto el cumplimiento de las normas establecidas posibilitaran alcanzar las características deseables entre sus integrantes; siendo los resultados según el rho de Spearman las correlaciones entre el clima institucional y desempeño docente (rho=0,537); con comportamiento institucional (rho $=0,957)$; con capacidad organizacional $(\mathrm{rho}=0,890)$ y dinámica institucional $(r h o=0,920)$. Asi también Saccsa (2010) indicó que, el clima en una institución escolar es determinante para contar con un ambiente que posibilite el desarrollo de aprendizajes de los educandos; y en donde los estilos comunicativos juegan un papel crucial para el logro del perfil en los educandos, es por ello que, la escuela debe promover una comunicación y la participación permanente proponiendo la práctica de valores éticos entre los miembros de la comunidad educativa; siendo los resultados obtenidos en la regresión logística se obtuvo un valor $\mathrm{R}=0,768 \mathrm{p}=0,000$; concluyéndose que el clima institucional afecta el desempeño de la escuela de manera ostensible y se debe de trabajar en este aspecto con miras a elevar el mejoramiento de las relaciones humanas en la comunidad educativa. Por consiguiente, Yopan, Palmero y Santos (2020), manifestaron que la cultura organizacional se considera en estos momentos en uno de los activos más significativos en una organización y las teorías que fortalecen este constructo se encuentra en los campos administrativos, organizacionales y comunicacionales; en ese sentido, la cultura organizacional se construye a través de procesos de interacción e interpretación, en esta argumentación se configura en una tarea que remarca complejidad y de carácter cualitativo.

\section{CONCLUSIONES}

\section{Primera}

Se logró determinar la relación entre Investigación Universitaria, Clima y Cultura organizacionales en la Universidad César Vallejo - sede Lima Este, siendo necesario desarrollar con un mayor grado de intensidad las actividades de investigación universitaria en su nivel formativo ya que constituye el soporte fundamental en el desarrollo profesional de los estudiantes y se debe impulsar a partir los primeros ciclos de estudio con niveles de compromiso y responsabilidad académica. 


\section{Segunda}

Se logró determinar la relación entre los procesos de enseñanza, el clima y la cultura organizacional, debido a que estas impactan directamente en el desarrollo de la práctica investigativa de los estudiantes universitarios, asi también potenciar con la aplicación de metodologías innovadoras que propendan alcanzar los objetivos educacionales en su proceso formativo de los estudiantes universitarios.

\section{Tercera}

Se logró determinar la relación entre los procesos de aprendizaje, el clima y la cultura organizacional, en ese sentido se tendrá que considerar los factores que inciden el proceso formativo que afectaran positivamente en el desarrollo de los proyectos de investigación en el entorno investigativo, entre los indicadores de mayor relevancia se observa el tiempo y los recursos académicos, la participación en el trabajo de campo, tanto los aspectos teóricos como los aspectos personales para concretizar el trabajo de investigación científica.

\section{Cuarta}

Se logró determinar la relación entre el desarrollo de proyectos de investigación, el clima y la cultura organizacional en lo que se observa que se revela una óptima predisposición por parte de los estudiantes en las actividades de campo y que se servirá de sustento para la investigación universitaria.

\section{REFERENCIAS}

Arias-Gómez, J., Villasís-Keever, M.A. y Miranda-Novales, M.G. (2016). El protocolo de investigación III: la población de estudio. Revista Alergia México, 63(2), 201-206. Recuperado de: https://www.redalyc.org/pdf/4867/486755023011.pdf

Bohórquez-Ordoñez, A.A. (2015). Actitud científica y logro de las competencias del curso de investigación en los estudiantes de Educación de la Universidad Nacional Mayor de San Marcos, 2015. (Tesis doctoral). Universidad Nacional de educación Enrique Guzmán y Valle. Recuperado de: https://repositorio.une.edu.pe/bitstream/handle/UNE/894/TD\%20CE\%20B694\%20 2015.pdf? sequence $=1 \&$ is Allowed $=\mathrm{y}$

Chiang, M., Núñez, A., y Martín, M. (2010). Relaciones entre el clima laboral y la satisfacción laboral. Madrid: Universidad Pontificia Comillas. Recuperado de: https://books.google.com.pe/books?id=v_sFY1XRFaIC\&printsec=frontcover\&sour

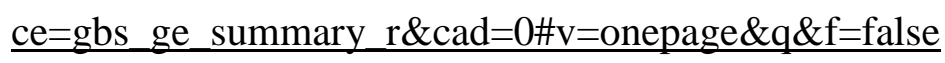


Chiavenato, I. (1999). Administración de Recursos Humanos. (5a ed.), México: McGraw Hill.

Dáher-Nader, J.E., Panunzio, A.P. y Hernández-Navarro, M.I. (2018). La investigación científica: una función universitaria a considerar en el contexto ecuatoriano. Edumecentro, 10(4): 1-14. Recuperado de: http://scielo.sld.cu/pdf/edu/v10n4/edu11418.pdf

Denison, D. (2006). Clima organizacional y gerencia: inductores del cambio organizacional, Revista de investigación y Postgrado, vol 21, Caracas: Universidad Experimental Libertador.

Duarte de Krummel, M. (2015). Importancia de la investigación científica en la vida universitaria. Revista Científica de la UCSA, 2(2): 3-5. Recuperado de: http://scielo.iics.una.py/pdf/ucsa/v2n2/v2n2a01.pdf

Fuentes, A. (2012). La cultura organizacional: Factor clave de éxito en las empresas del Siglo XX1. (Tesis de grado). Universidad de Cartagena. Cartagena de Indias, Colombia. Recuperado de: http://190.242.62.234:8080/jspui/bitstream/11227/1856/1/381-\%20ensayo\%20\%201a\%20cultura\%20organizacional $\% 20$ factor\%20clave $\% 20 \mathrm{de} \% 20 \%$ c3\%89xito $\% 2$ n\%20las\%20empresas\%20de1\%20siglo\%20xxi.pdf

Guevara-Pazmiño, X. (2018). Clima organizacional Nivel de satisfacción en la Unidad Educativa Particular La Dolorosa. (Tesis de maestría). Universidad Andina Simón Bolívar. Recuperado de: https://repositorio.uasb.edu.ec/bitstream/10644/6169/1/T2597-MIE-GuevaraClima.pdf

Martín-Bris, M. (1999). Clima de trabajo y participación en la organización y funcionamiento de los centros de educación. Madrid: Universidad de Alcalá-MEC.

Méndez, C. (2006). Clima organizacional en Colombia. El IMCOC: Un método de análisis para su intervención. Colección de lecciones de administración. Bogotá: Universidad del Rosario

Ñaupas, H. (2009). Metodología de la investigación científica y asesoramiento de tesis. Lima, Perú: Gráfica Retai S.A.C

Peniche, R. (2012). Las instituciones educativas como organismos micropolíticos: Estudio cualitativo en una Institución de nivel superior. (Tesis doctoral). Universidad de Granada. Granda, España. Recuperado de https://hera.ugr.es/tesisugr/21876605.pdf 
Pérez-Huamán, Y.M. (2012). Relación entre el clima institucional y desempeño docente en instituciones educativas de la Red No 1 Pachacútec - Ventanilla. (Tesis de maestría). Universidad San Ignacio de Loyola. Recuperado de: https://repositorio.usil.edu.pe/server/api/core/bitstreams/7aafd572-d054-40f7-a4b7$\underline{\mathrm{b} 90 \mathrm{f} 90 \mathrm{~b} 4 \mathrm{e} 13 \mathrm{a} / \text { content }}$

Piscoya, L. (1987). Investigación científica y educacional. Lima, Perú: Amaru Editores.

Ríos-Marín, J. G. (2013). La investigación científica como medio para adquirir conocimiento significativo. Revista educación y desarrollo social. 7(1), 108-115. Recuperado de: https://revistas.unimilitar.edu.co/index.php/reds/article/view/738

Saccsa, J. (2010). Relación entre clima institucional y el desempeño académico de los docentes de los Centros de Educación Básica Alternativa (CEBAs) del distrito de San Martín de Porres. (Tesis de maestría). Universidad Nacional Mayor de San Marcos. Perú. Recuperado de: http://cybertesis.unmsm.edu.pe/bitstream/handle/cybertesis/2405/Saccsa_cj.pdf?sequ ence $=1 \&$ is Allowed $=\mathrm{y}$

Sánchez-Carlessi, H. y Reyes-Meza, C. (2015). Metodología y diseños en la investigación científica. Perú.

Támara-Mautino, C.P. (2018). Clima organizacional y calidad del servicio educativo de los docentes de la I.E.PUB. "Augusto Salazar Bondy" - Chancay 2017. (Tesis de maestría). Universidad César Vallejo, Perú. Recuperado de: https://repositorio.ucv.edu.pe/bitstream/handle/20.500.12692/14465/T\%C3\%A1mara MCP.pdf? sequence $=1$ \&isAllowed $=\mathrm{y}$

Vargas Medina, V.A. (2020). Actitud hacia la investigación científica de docentes universitarios de Huancayo - 2017. (Tesis doctoral). Universidad Nacional del Centro del Perú. Recuperado de: https://repositorio.uncp.edu.pe/bitstream/handle/20.500.12894/6608/T010_41303971 D.pdf?sequence $=1$

Yopan-Fajardo, J.L., Palmero-Gómez, N. y Santos-Mejía, J.R. (2020). Cultura organizacional. Controversias y concurrencias latinoamericanas, 11(20), 263-281. Recuperado de: https://www.redalyc.org/articulo.oa?id=588663787016 